\title{
Synthesis and Crystal Structure of Ethyl Zinc Chloride
}

Antonio Guerrero, David L. Hughes, Manfred Bochmann.*

\section{Supporting Information}

Tables of crystallographic and refinement data for $\mathrm{EtZnCl}$.

Crystal and structure refinement data for ZnEtCl

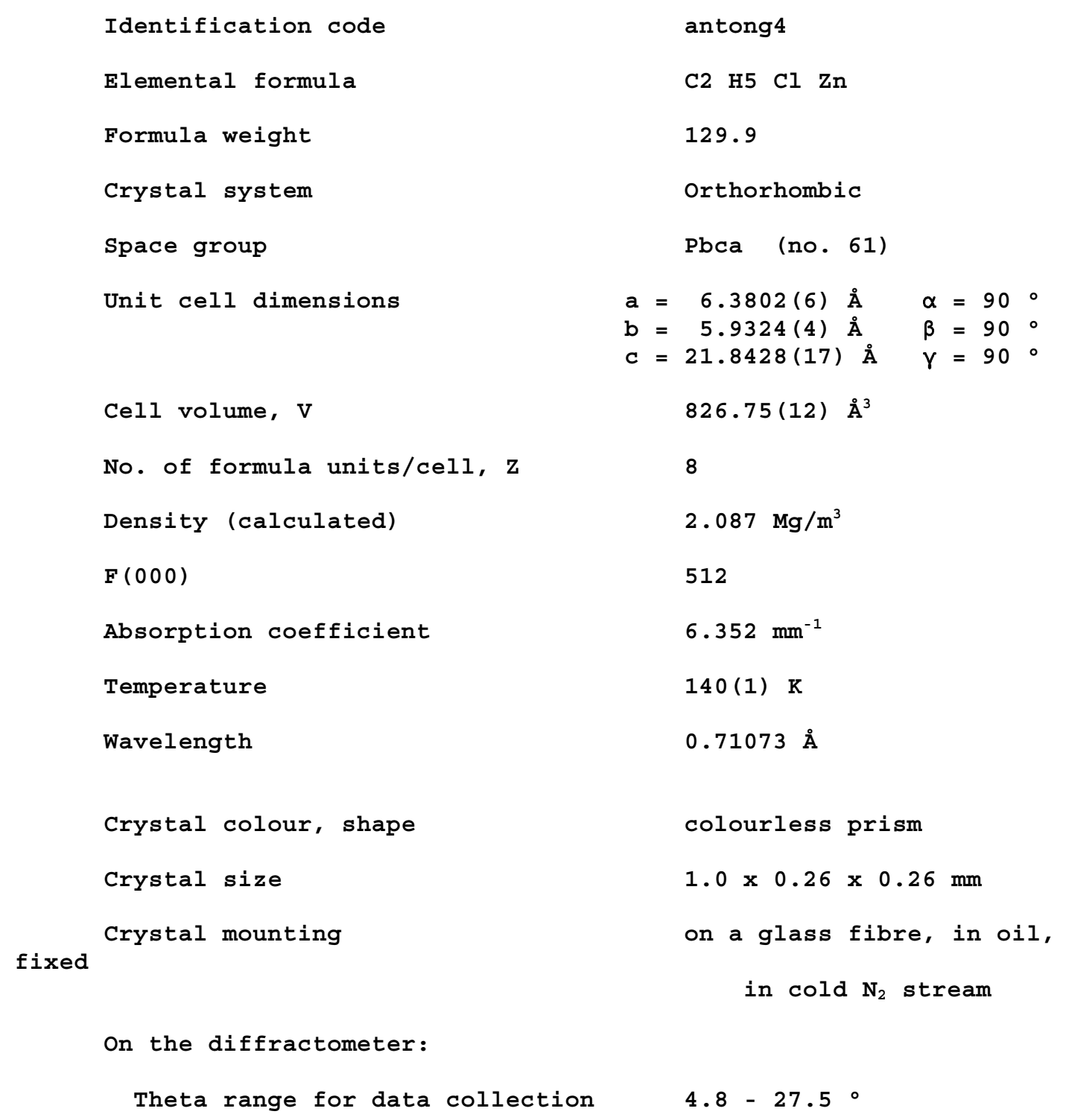


Index ranges for $h, k, 1$

Completeness to theta $=27.5$

Absorption correction

equivalents

Max. and min. transmission

Total no. of reflections measured (not including absences)

No. of unique reflections $0.065)$

949 (Rint for equivalents =

No. of 'observed' reflections $\left(I>2 \sigma_{I}\right) 926$

Structure determined by: direct methods, in SHELXS

SHELXL

Refinement: $\quad$ Full-matrix least-squares on $\mathrm{F}^{2}$, in

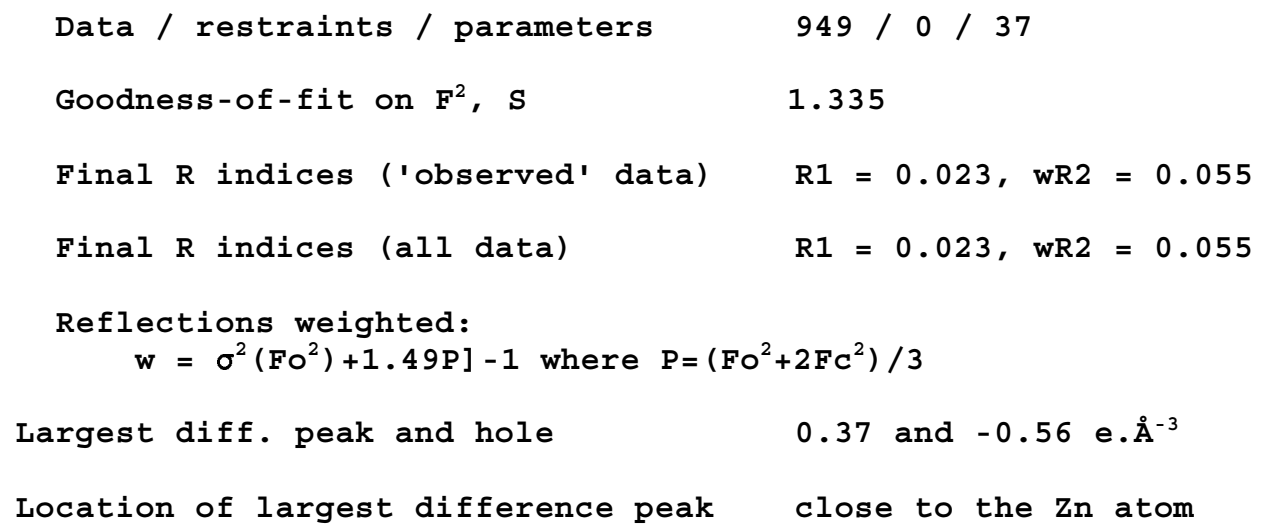


Table 1. Atomic coordinates (x $10^{4}$ ) and equivalent isotropic displacement parameters $\left(\AA^{2} \times 10^{4}\right)$. U(eq) is defined as one third of the trace of the orthogonalized Uij tensor. E.s.ds are in parentheses.

\begin{tabular}{lccll}
\hline & & & & \\
& & $y$ & & \\
& & & & \\
\hline $\mathrm{Zn}$ & $316.5(5)$ & $1765.2(5)$ & $6896.3(1)$ & $192.0(11)$ \\
$\mathrm{Cl}$ & $4148.2(9)$ & $907.2(9)$ & $7111.1(2)$ & $123.1(13)$ \\
$\mathrm{C}(1)$ & $-561(4)$ & $1062(5)$ & $6064.5(11)$ & $197(5)$ \\
$\mathrm{C}(2)$ & $496(5)$ & $2537(5)$ & $5577.7(12)$ & $275(7)$ \\
\hline
\end{tabular}

Table 2. Molecular dimensions. Bond lengths are in Angstroms, angles in degrees. E.s.ds are in parentheses.

(a) About the zinc and chloride atoms

$\begin{array}{llll}\mathrm{Zn}-\mathrm{Cl} & 2.5408(7) & \mathrm{Zn}-\mathrm{Cl} & 2.5248(6) \\ \mathrm{Zn}-\mathrm{Cl} 1^{\mathrm{a}} & 2.3486(6) & \mathrm{Zn}-\mathrm{C}(1) & 1.946(2) \\ \mathrm{Cl} & & & \\ \mathrm{Cl}-\mathrm{Zn}-\mathrm{Cl} & 95.250(17) & \mathrm{C}(1)-\mathrm{Zn}-\mathrm{Cl} & 113.96(8) \\ \mathrm{Cl} 1^{\mathrm{a}}-\mathrm{Zn}-\mathrm{Cl} l^{\mathrm{b}} & 91.748(18) & \mathrm{C}(1)-\mathrm{Zn}-\mathrm{Cl} 1^{\mathrm{a}} & 136.39(8) \\ \mathrm{Zn}^{\mathrm{c}}-\mathrm{Cl}-\mathrm{Zn} & 94.722(18) & \mathrm{C}(1)-\mathrm{Zn}-\mathrm{Cl} 1^{\mathrm{b}} & 114.90(8) \\ \mathrm{Zn}^{\mathrm{d}}-\mathrm{Cl}-\mathrm{Zn} & 115.62(2) & \mathrm{Zn}^{\mathrm{c}}-\mathrm{Cl}-\mathrm{Zn}^{\mathrm{d}} & 109.85(2)\end{array}$

(b) In the methyl group

$C(1)-C(2) \quad 1.533(4) \quad C(2)-C(1)-\mathrm{Zn} \quad 113.48(18)$

Symmetry transformations used to generate equivalent atoms:

$a: x-1 / 2, y, 1 \frac{1}{2}-z \quad b: 1 / 2-x, y+1 / 2, z$

c : $x+1 / 2, y, 1 \frac{1}{2}-\mathbf{z} \quad \mathrm{d}: 1 / 2-x, y-1 / 2, z$ 
Table 3. Anisotropic displacement parameters $\left(\AA^{2} \times 10^{4}\right)$ for the expression:

$$
\exp \left\{-2 \pi^{2}\left(h^{2} a *^{2} U_{11}+\ldots+2 h k a * b * U_{12}\right)\right\}
$$

E.s.ds are in parentheses.

\begin{tabular}{cccccrr}
\hline & $\mathrm{U}_{11}$ & $\mathrm{U}_{22}$ & $\mathrm{U}_{33}$ & $\mathrm{U}_{23}$ & $\mathrm{U}_{13}$ & $\mathrm{U}_{12}$ \\
\hline $\mathrm{Zn}$ & $236(2)$ & $217(2)$ & $123(2)$ & $-20.9(11)$ & $-25.2(11)$ & - \\
$12.4(12)$ & & & & & & \\
$\mathrm{Cl}$ & $132(3)$ & $102(3)$ & $136(3)$ & $-9(2)$ & $-22(2)$ & $4(2)$ \\
$\mathrm{C}(1)$ & $217(14)$ & $224(13)$ & $152(11)$ & $-18(10)$ & $-43(10)$ & $-32(11)$ \\
$\mathrm{C}(2)$ & $357(19)$ & $282(16)$ & $185(13)$ & $38(12)$ & $23(11)$ & $18(13)$
\end{tabular}

Table 4. Hydrogen coordinates ( $x$ 104) and isotropic displacement parameters $\left(\AA^{2} \times 10^{3}\right)$. All hydrogen atoms were included in idealised positions with U(iso)'s set at $1.2 * U(e q)$

or, for the methyl group, $1.5 * U(e q)$ of the parent carbon

atom.

\begin{tabular}{lrrrr}
\hline & $\mathbf{x}$ & $\mathbf{y}$ & $\mathbf{z}$ & U(iso) \\
\hline $\mathrm{H}(1 \mathrm{a})$ & -243 & -506 & 5980 & 24 \\
$\mathrm{H}(1 \mathrm{~b})$ & -2067 & 1250 & 6035 & 24 \\
$\mathrm{H}(2 \mathrm{a})$ & 4 & 2107 & 5179 & 41 \\
$\mathrm{H}(2 \mathrm{~b})$ & 1988 & 2338 & 5598 & 41 \\
H (2c) & 158 & 4091 & 5651 & 41 \\
\hline
\end{tabular}

Table 5. Torsion angles, in degrees. E.s.ds are in parentheses.

$\begin{array}{lc}\mathrm{C}(1)-\mathrm{Zn}-\mathrm{Cl}-\mathrm{Zn}^{\mathrm{c}} & 179.20(9) \\ \mathrm{C} 1^{\mathrm{a}}-\mathrm{Zn}-\mathrm{Cl}-\mathrm{Zn}^{\mathrm{c}} & -34.02(4) \\ \mathrm{Cl} 1^{\mathrm{b}}-\mathrm{Zn}-\mathrm{Cl}-\mathrm{Zn}^{\mathrm{c}} & 60.88(3) \\ \mathrm{C}(1)-\mathrm{Zn}-\mathrm{Cl}-\mathrm{Zn}^{\mathrm{d}} & -58.16(9) \\ \mathrm{Cl} 1^{\mathrm{a}}-\mathrm{Zn}-\mathrm{Cl}-\mathrm{Zn}^{\mathrm{d}} & 88.61(2) \\ \mathrm{Cl} 1^{\mathrm{b}}-\mathrm{Zn}-\mathrm{Cl}-\mathrm{Zn}^{\mathrm{d}} & -176.482(9) \\ \mathrm{Cl} 1^{\mathrm{a}}-\mathrm{Zn}-\mathrm{C}(1)-\mathrm{C}(2) & 166.41(15) \\ \mathrm{Cl} 1^{\mathrm{b}}-\mathrm{Zn}-\mathrm{C}(1)-\mathrm{C}(2) & 38.2(2) \\ \mathrm{Cl}-\mathrm{Zn}-\mathrm{C}(1)-\mathrm{C}(2) & -65.9(2)\end{array}$

Symmetry transformations used to generate equivalent atoms:

$a: x-1 / 2, y, 1 \frac{1}{2}-z \quad b: 1 / 2-x, y+1 / 2, z$

c : $x+1 / 2, y, 1 \frac{1}{2}-\mathbf{z} \quad \mathrm{d}: 1 / 2-x, y-1 / 2, z$ 